Pacific Journal of Mathematics

FLING EXPASSON I REAL FLO 


\title{
MODELLING EXPANSION IN REAL FLOWS
}

\author{
H. B. Keynes and M. Sears \\ Dedicated to the memory of Rufus Bowen
}

\begin{abstract}
We show that any real flow without fixed points is the homomorphic image of a suspension of the shift on a bisequence space and the homomorphism is one-to-one between invariant residual sets. If the original flow is onedimensional this homomorphism is an isomorphism. We then use this model of a real flow to lift $\mathscr{F}$-expansiveness for any class $\mathscr{F}$ of continuous functions from the reals into the reals fixing zero, and thus generalize the results of Bowen and Walters [2]. Various other properties of the suspension model are discussed.
\end{abstract}

o. Introduction. In [2] Bowen and Walters introduced the concept of expansiveness for real flows relative to the class $\mathscr{C}$ of all continuous functions from the reals into the reals which fix zero. In [5] this concept was extended to arbitrary transformation groups and to arbitrary classes $\mathscr{F}$ of continuous functions from the acting group into itself which fixes the group identity. It is well-known (see [4]) that any expansive discrete flow can be lifted to a subshift and the authors of [2] managed to obtain an analogous result for real flows: every $\mathscr{C}$-expansive real flow can be lifted to the suspension of a shift on a symbol space. Results of this type are also obtained for $\mathscr{F}$-expansive discrete flows in [5] but no results were given for other transformation groups. The main direction of this paper is to extend the methods and results of [2] to show that every fixed point free $\mathscr{F}$-expansive real flow can be lifted to a suspension of a shift on a bisequence space (of course, not a finite symbol space in general) which is also $\mathscr{F}$-expansive. This generalizes the Bowen and Walters result and also covers situations where real flows can not be $\mathscr{C}$-expansive but are expansive for subclasses $\mathscr{F}$, such as certain real flows on a 2-torus (see [5] and [3] for details).

Since the model that we obtain is independent of any expansive properties of the original flow, we also obtain a result of interest in its own right concerning real flows and suspensions. Birkhoff [1] pointed out that certain dynamical systems have a global cross section and can thus be regarded as real suspensions of discrete flows. Schwartzman [6] showed that for real flows on compact metric spaces, the property of possessing a global section was equivalent to the flow being a suspension over a discrete flow on that 
global section and the local section approach was used by Bowen and Walters to obtain their suspension model under the assumption that the original flow was real-expansive. We will show that every real flow without fixed points can be modelled in this way. Precisely if $(X, \boldsymbol{R})$ is such a flow, then there is a space of bisequences $\hat{X}$ and a continuous real-valued function $\hat{t}$ on $\hat{X}$ such that $(X, R)$ is a homomorphic image of the suspension of $(\hat{X}, \sigma)$ (where $\sigma$ is the shift on $\hat{X}$ ) by $\hat{t}$ and the homomorphism is one-to-one between invariant residual sets. Furthermore there is a nontrivial subshift $\left(\hat{X}(\mathscr{S}), \sigma_{0}\right)$ on a finite symbol space associated with $X$, and various conditions (including $\mathscr{C}$-expansive, but also others) imply that $(\hat{X}, \sigma)$ and $\left(\hat{X}(\mathscr{S}), \sigma_{0}\right)$ are isomorphic. Finally, if $X$ is one-dimensional, then $(X, R)$ is (isomorphic to) this suspension. We then show that this suspension construction is a good model from the point of view of $\mathscr{F}$-expansiveness.

1. Preliminaries. We begin by giving some definitions and notation for real flows. All flows will be on a compact metric space and if $x \in X$, the action of $t \in \boldsymbol{R}$ on $x$ will be denoted by $x t$.

Definition 1.1. Given a set $\mathscr{F}$ of continuous functions $f: R \rightarrow$ $R$ such that $f(0)=0$, a flow $(X, \boldsymbol{R})$ is called $\mathscr{F}$-expansive if for each $\varepsilon>0$ there is a $\delta>0$ such that if $f \in \mathscr{F}$ and $x, y \in X$ with $x \notin y(-\varepsilon, \varepsilon)$ then for some $t \in R, d(x t, y f(t))>\delta$.

$\mathscr{F}$-expansive flows are discussed in [2] and [5].

DEFINITION 1.2. Let $X$ be a compact metric space and $\psi$ a homeomorphism from $X$ onto itself. Let $g$ be a positive continuous real-valued function on $X$. Then the real suspension of $(X, \psi)$ by $g$ is the real flow constructed as follows. Form the quotient space $X \times \boldsymbol{R} / \sim$ where $\sim$ is the equivalence relation $(x, g(x)) \sim(\psi x, 0)$. The action is defined on this space by $(x, t) s=\left(\psi^{n} x, u\right)$ where $t+s=$ $u+x_{0} \sum_{i=0}^{n-1} g\left(\psi^{i} x\right)$ and $0 \leqq u<g\left(\psi^{n} x\right)$. Locally the metric on $X \times \boldsymbol{R} / \sim$ can be taken as equivalent to the sum of the distances along the orbit and across orbits, i.e., for $t, s$ suitably small, $D[(x, t),(y, s)]$ is small precisely when both $|t-s|$ and $\min \{d(x, y), d(\psi x, \psi y)\}$ are small, where $d$ is a metric on $X$. The suspension flow is denoted $\left(X_{g}, \boldsymbol{R}\right)$.

A complete discussion of suspensions is given in [2].

Definition 1.3. Let $(X, \boldsymbol{R})$ be a real flow. A local cross section (or simply section) at a point $x \in X$ is a closed set $S \subset X$ with $x \in S$ and such that for some $\delta>0, S \cap S(0, \delta]=S \cap S[-\delta, 0)=\phi . \quad \delta$ is referred to as a section time for $S$. 
It is well-known (see for example [8]) that if $(X, R)$ has no fixed points then for any $x \in X$, there is a section containing $x$. If $S$ is a section, $S \times[-\delta, \delta]$ is homeomorphic to $S[-\delta, \delta]$. Further if we define $S^{*}=\operatorname{Int}(S(-\delta, \delta)) \cap S$ then $S^{*}(-r, r)$ is open in $X(r>0)$, $S^{*}$ is open in $S$, and given $x$, we can assume $x \in S^{*}$. We can, in fact, always choose sections such that $S^{*}$ is dense in $S$ by the following lemma, whose proof is routine and thus omitted.

Lemma 1.4. Let $S$ be a section and let $A=\overline{S^{*}}$. Then $A$ is a section for any point $x \in S^{*}$ and $A^{*}=A$.

Theorem 1.5. (Bowen and Walters [2].) There is a $\zeta>0$ so that the following holds: For each $\alpha>0$ there is a finite family $\mathscr{S}$ of pairwise disjoint local sections of time $\zeta$ and diameter at most $\alpha$ such that $X=Y[-\alpha, 0]=Y[0, \alpha]$ where $Y=\bigcup_{S \epsilon,} S$. Further, we may assume that $\overline{S^{*}}=S(S \in \mathscr{S})$, and that if $X$ is one-dimensional, $S=S^{*}(S \in \mathscr{S})$.

Proof. This is Lemma 7 of [2] with the extra condition that $\overline{S^{*}}=S$, which follows from 1.4 and minor changes in the proof.

2. Lifting real flows to suspensions. Let $(X, R)$ be a real flow without fixed points. Choose $\zeta$ as in Theorem 1.5 and $\mathscr{S}$ and $\alpha$ satisfying the conditions of that theorem and also $2 \alpha<\zeta$. Let $Y=\mathrm{U}_{S \in \mathcal{L}} S$ have its topology as a subspace of $X$ and let $\rho$ be the minimum flow time between sections i.e., if $x \in Y$ then $x(0, \rho) \cap Y=$ $x(-\rho, 0) \cap Y=\phi$. Now if $x \in Y$ define the first return time $t(x)$ by the smallest positive time such that $x t(x) \in Y$. Note that since $X=$ $Y[0, \alpha]$ such a time always exists and clearly $t: Y \rightarrow[\rho, \alpha]$. Now define a function $\varphi: Y \rightarrow Y$ by $\varphi(x)$ being the first return of $x$ to $Y$ (i.e., the first point of the positive semi-orbit of $x$ which intersects $Y)$. Thus $\varphi(x)=x t(x)$. Note that $t$ and $\varphi$ are well-defined but will not be continuous in general. The next few results are concerned with the basic continuity properties of these maps.

LEMma 2.1. $\varphi$ is a one-to-one map from $Y$ onto itself. If $y \in$ $Y$ such that $\varphi(y) \in S^{*}$ for some $S \in \mathscr{S}$, then $\phi$ is continuous at $y$. The latter result holds for $\varphi^{-1}$, and for first return time $t(\cdot)$.

Proof. That $\varphi$ is one-to-one and onto (hence $\varphi^{-1}$ exists) is routine. Now suppose $y \in Y$ and $\varphi(y) \in S^{*}$ for some $S \in \mathscr{S}$. Choose $\delta>0$ with $\delta<\rho / 2$ and let $N$ be a neighborhood of $\varphi(y)$ contained in $S^{*}$. By the continuity of the action we can find a neighbor- 
hood $U(y)$ of $y$ such that $U(y) t(y) \subset N(-\delta, \delta)$. Now if $x \in U(y)$, then $x t(y) \in N(-\delta, \delta)$ and so $x(t(y)+\eta) \in N$ for some $\eta$ with $|\eta|<\delta$. Also since $y(0, t(y)) \cap Y=\phi$, for all $\varepsilon>0$ with $\varepsilon<\rho / 2$ we can find a neighborhood $V(y)$ of $y$ such that $V(y)(\varepsilon, t(y)-\varepsilon) \cap Y=\phi$. Now if $x \in V(y) \cap U(y) \cap Y$ then $x(0, \varepsilon] \cap Y=x(\varepsilon, t(y)-\varepsilon) \cap Y=\phi$ and $x(t(y)+$ $\eta) \in N$ for some $\eta$ with $|\eta|<\delta$ so $x[t(y)-\varepsilon, t(y)+\eta) \cap Y=\phi$. Thus $t(x)=t(y)+\eta$ and so $x t(x) \in N$ and hence $\varphi(V(y) \cap U(y) \cap Y) \subset N$. A similar argument can be used for $\varphi^{-1}$, and first return time $t(\cdot)$.

Let $A=\cup\left\{S-S^{*} ; S \in \mathscr{S}\right\}$. Simple examples show that in general $\varphi(A)$ is not closed. However, we have:

Lemma 2.2. For any integer $m \geqq 0, \bigcup_{k=0}^{m} \varphi^{k}(A)$ is a closed nowhere dense set in $Y$.

Proof. We first show that $\bigcap_{k=0}^{m} \varphi^{k}(\mathscr{C}(A))$ is open. If $x \in$ $\bigcap_{k=0}^{m} \varphi^{k}(\mathscr{C}(A))$ then $\varphi^{-l} x \in \mathscr{C}(A), 0 \leqq l \leqq m$, and applying 2.1, we have that $\varphi^{-l}$ is continuous at $x, 0 \leqq l \leqq m$. Since $\mathscr{C}(A)$ is open, there exists a neighborhood $U$ of $x$ with $\mathscr{P}^{-l}(U) \subset \mathscr{C}(A), 0 \leqq l \leqq m$. Thus, $U \subset \bigcap_{k=0}^{m} \phi^{k}(\mathscr{C}(A))$.

We show $\bigcup_{k=0}^{m} \varphi^{k}(A)$ is nowhere dense by induction on $m$. Clearly $m=0$ holds, and suppose it holds for $m-1$. If $\bigcup_{k=0}^{m} \phi^{k}(A)$ has interior, then $A$ nowhere dense implies that there must be an open set, $U$ say, with $U \subset \bigcup_{k=1}^{m} \varphi^{k}(A)$ and $U \subset \mathscr{C}(A)$. Now by Lemma 2.1 we can find an open set $V$ in $Y$ with $\varphi(V) \subset U$. But then $V=\varphi^{-1} \varphi(V) \subset \varphi^{-1} U \subset \bigcup_{k=0}^{m-1} \varphi^{k}(A)$, contradicting the induction hypothesis. This completes the proof.

Corollary 2.3. Let $Z=\mathscr{C}\left(\bigcup_{k=-\infty}^{\infty} \varphi^{k}(A)\right)$. Then $Z$ is a dense $G_{\delta}$ set in $Y$.

Proof. Clearly Lemma 2.2. applies to $\varphi^{-1}$ also.

CoRollary 2.4. If $x \in Z$, then $\varphi$ and $\varphi^{-1}$ are continuous along the -orbit of $x$.

Proof. For every $n, \varphi^{n}(x) \notin A$, and the result follows by Lemma 2.1 .

We can now construct the discrete flow which will be the base for the suspension. For each integer $k$, let $X_{k}=Y$ and form $\Omega=$ $\Pi_{x=-\infty}^{\infty} X_{k}$, the compact metric space of all bisequences whose entries are elements of $Y$. Let $\sigma$ be the shift map i.e., $\sigma(x)_{i}=x_{i+1}$ for $x \in \Omega$. Let $\hat{Z}=\left\{\left(\varphi^{k}(x)\right)_{-\infty}^{\infty} ; x \in Z\right\} \subset \Omega$, and $\hat{X}=\operatorname{cls} \hat{Z}$. Define $\hat{\pi}: \hat{X} \rightarrow Y$ by $\hat{\pi}(x)=x_{0}$, i.e., projection onto the zeroth coordinate. 
The following lemma summarizes several properties of the discrete flow $(\hat{X}, \sigma)$.

Lemma 2.5. (i) $(\hat{X}, \sigma)$ is a discrete flow.

(ii) $\hat{\pi}$ is a continuous map from $\hat{X}$ onto $Y$.

(iii) If $\phi$ is continuous, $\hat{\pi}$ is an isomorphism between $(\hat{X}, \sigma)$ and $(Y, \varphi)$.

(iv) If $X$ is one-dimensional, we can choose $\mathscr{S}$ so that $(\hat{X}, \sigma)$ is isomorphic to $(Y, \varphi)$.

(v) If $x \in \hat{X}$, there is a $t \in[\rho, \alpha]$ such that $x_{0} t=x_{1}$.

(vi) The function $\hat{t}: \hat{X} \rightarrow[\rho, \alpha]$ defined by setting $\hat{t}(x)$ to be the first time $t>0$ such that $x_{0} t=x_{1},(x \in \hat{X})$ is continuous.

(vii) If $x \in Y, \hat{\pi}^{-1}(x)$ is the single point $\left(\phi^{k}(x)\right)_{-\infty}^{\infty}$ if $\phi^{k}$ is continuous at $x$ for every $k$.

(viii) $\hat{\pi}$ is a one-to-one map between invariant residual sets which contain $Z$ and $\hat{Z}$ respectively.

Proofs. (i) If $x \in Z$, then $\varphi^{k} x \in Z$ for any $k$ and thus $\hat{Z}$ is invariant under $\sigma$, which means that $\hat{X}$ is invariant under $\sigma$.

(ii) Since $\hat{\pi}$ is continuous and $\hat{\pi}(\hat{Z})=Z, \hat{\pi}(\hat{X})=\operatorname{cls} Z=Y$.

(iii) If $\varphi$ is continuous, i.e., $(Y, \varphi)$ is a discrete flow, it is routine to verify that $\hat{X}=\left\{\left(\varphi^{k} x\right)_{-\infty}^{\infty} \mid x \in Y\right\}$, and that $\hat{\pi}$ is a homeomorphism onto. Since $\varphi \hat{\pi}(x)=\varphi\left(x_{0}\right)=\hat{\pi}(\sigma x)$, for $x \in \hat{X}, \hat{\pi}$ is an isomorphism.

(iv) By Theorem 1.2 we can choose $\mathscr{S}$ such that $S^{*}=S$ for every $S \in \mathscr{S}$. Then $\varphi$ is continuous by Lemma 2.1 and the result follows by (iii).

(v) Let $x \in \hat{X}$ and suppose $\left\{z^{(n)}\right\}$ is a sequence in $\hat{Z}$ with $z^{(n)} \rightarrow x$. Then $z_{0}^{(n)} \rightarrow x_{0}$ and $z_{1}^{(n)} \rightarrow x_{1}$ so $z_{0}^{(n)} t\left(z_{0}^{(n)}\right) \rightarrow x_{1}$. By choosing a subsequence if necessary we can assume that $t\left(z_{0}^{(n)}\right)$ converges to some point, $s$ say, with $\rho \leqq s \leqq \alpha$. Now the continuity of the action gives $x_{0} s=x_{1}$ as required.

(vi) Let $x \in \hat{X}$ and let $\left\{y^{(n)}\right\}$ be a sequence in $\hat{X}$ with $y^{(n)} \rightarrow x$. Note that $y_{1}^{(n)}=y_{0}^{(n)} \hat{t}\left(y^{(n)}\right)$ for each $n>0$ and $x_{1}=x_{0} \hat{t}(x)$. Choose a subsequence $\left\{y^{\left(n_{2}\right)}\right\}$ such that $\hat{t}\left(y^{\left(n_{2}\right)}\right)$ converges to some limit, $s$ say. Then clearly $x_{0} \hat{t}(x)=x_{0} s$. Now $x_{0}(\hat{t}(x)-s)=x_{0}$ and since $|\hat{t}(x)-s| \leqq$ $2 \alpha<\zeta, \hat{t}(x)=s$. Thus we have a subsequence $\left\{y^{\left(n_{i}\right)}\right\}$ with $\hat{t}\left(y^{\left(n_{i}\right)}\right) \rightarrow$ $\hat{t}(x)$.

(vii) Suppose $\varphi^{k}$ is continuous at $x$ for every $k$, and suppose $w \in \hat{X}$ with $\hat{\pi}(w)=x$. Pick a sequence $\left\{z^{(n)}\right\}$ in $\hat{Z}$ with $z^{(n)} \rightarrow w$. Then $w_{0}=x$ and for every $k, \varphi^{k}\left(z_{0}^{(n)}\right)=z_{k}^{(n)} \rightarrow w_{k}$. But $\varphi^{k}\left(z_{0}^{(n)}\right) \rightarrow \varphi^{k}(x)$ by continuity, and so $w=\left(\varphi^{k}(x)\right)_{-\infty}^{\infty}$.

(viii) We consider the invariant set $V=\left\{x \in Y: \varphi\right.$ and $\varphi^{-1}$ continuous along the orbit of $x\}$. Then $Z \subset V$ by 2.4, and $\hat{\pi} \mid \hat{\pi}^{-1}(V)$ : 
$\hat{\pi}^{-1}(V) \rightarrow V$ is one-one by (vii). To complete the proof, we need only show that $\hat{Z}$ is residual in $\hat{X}$. But $Z=\bigcap_{m=-\infty}^{\infty} \mathscr{C}\left(\bigcup_{k=0}^{m} \varphi^{k}(A)\right)$ implies $\hat{Z}=\hat{\pi}^{-1}(\boldsymbol{Z})=\bigcap_{m=-\infty}^{\infty} \hat{\pi}^{-1}\left(\mathscr{C}\left(\bigcup_{k=0}^{m} \varphi^{k}(A)\right)\right)$ and $\hat{\pi}^{-1}\left(\mathscr{C}\left(\bigcup_{k=0}^{m} \varphi^{k}(A)\right)\right)$ is open and dense by 2.2 .

Several comments are in order concerning 2.5. Points in $\hat{X}-\hat{Z}$ have the form $x=\left(\mathscr{P}^{n_{k}}\left(x_{0}\right)\right)_{-\infty}^{\infty}$ by (v). Simple examples show that the sequence $\left\{n_{k}\right\}$ need not be increasing (i.e., in the case of periodic points one need not pick up points on the $\varphi$-orbit in correct reduced order), or that every point in the $\varphi$-orbit of $x_{0}$ appears in this representation. However, one can show that if $\varphi^{l}\left(x_{0}\right) \notin A$, i.e., $\varphi^{l}\left(x_{0}\right)$ is not a boundary point, then for some $k, n_{k}=l$. Since the proof is not germane to the rest of the paper, we shall omit it. Moreover, examples show that in general $\hat{\pi}$ is not an equivariant map from $(\hat{X}, \sigma)$ to $(Y, \varphi)$, and $\hat{\pi}^{-1}(x)$ may be a singleton even if $\varphi$ is discontinuous at $x$. In fact, one can have $\hat{X}$ homeomorphic to $Y$ for discontinuous $\varphi$. However, by forming the suspension $\left(\hat{X}_{\hat{t}}, \boldsymbol{R}\right)$ for $(\hat{X}, \sigma)$ with $\hat{t}$ as in (vi), then $\hat{\pi}$ does induce an equivariant map $\pi$ from $\left(\hat{X}_{\hat{t}}, \boldsymbol{R}\right)$ to $(X, \boldsymbol{R})$, as we now proceed to show. Again, one should note that even if $\hat{\pi}$ is a homeomorphism, $\pi$ need not be an isomorphism.

THEOREM 2.6. Let $(X, \boldsymbol{R})$ be a real flow without fixed points. Then $(X, \boldsymbol{R})$ is a quotient of the flow $\left(\hat{X}_{\hat{t}}, \boldsymbol{R}\right)$ and the natural quotient map is one-to-one between invariant residual subsets. Furthermore, if $X$ is one-dimensional, then $(X, R)$ is isomorphic to $\left(\hat{X}_{\hat{t}}, \boldsymbol{R}\right)$ for suitable choices of $\mathscr{S}$.

Proof. We map $\left(\hat{X}_{\hat{t}}, \boldsymbol{R}\right)$ to $(X, \boldsymbol{R})$ by a map $\pi$ defined by $\pi(x, s)=\hat{\pi}(x) s$ for any $(x, s) \in\left(\hat{X}_{\hat{t}}, \boldsymbol{R}\right)$ (i.e., $x \in \hat{X}$ and $\left.0 \leqq s<\hat{t}(x)\right)$. Note that since $(x, s) \rightarrow(\hat{\pi}(x), s)$ is continuous and $(\hat{\pi}(x), s) \rightarrow \hat{\pi}(x) s$ is continuous, the map $\pi$ is continuous and obviously onto.

To show that $\pi$ is equivariant we want $\pi[(x, s) t]=[\pi(x, s)] t$. Let $(x, s) \in \hat{X}_{\hat{t}}$ and set $s+t=\sum_{j=0}^{n-1} \hat{t}\left(\sigma^{j} x\right)+u$ where $0 \leqq u<\hat{t}\left(\sigma^{n} x\right)$. (Interpret the sum as zero if $n=0$.) Now $(x, s) t=\left(\sigma^{n} x, u\right)$ and so $\pi[(x, s) t]=\hat{\pi}\left(\sigma^{n} x\right) u=x_{n} u$ and $[\pi(x, s)] t=\hat{\pi}(x)(s+t)=x_{0}(s+t)=$ $x_{0}\left(\sum_{j=0}^{n-1} \hat{t}\left(\sigma^{j} x\right)\right) u=x_{n} u$ also.

Clearly, $A[-\alpha, \alpha]$ is nowhere dense and closed so that $A \boldsymbol{R}=$ $\mathrm{U}_{n=-\infty}^{\infty}(A[-\alpha, \alpha])(n \alpha)$ is a first category set in $X$. Note that this set contains $\mathscr{C} Z$, and let $W$ be its complement. Then $W$ is an invariant dense $G_{\delta}$ set in $X$. We only need to check that $\pi$ is oneto-one as a map from $\pi^{-1}(W)$ to $W$ and that $\pi^{-1}(W)$ is dense. First note that one can show that $W=Z \boldsymbol{R}$. Now let $(x, t)$ and $(y, s)$ be points in $\left(\hat{X}_{\hat{t}}, \boldsymbol{R}\right)$ with $\pi(x, t)=\pi(y, s) \in W$. Then $\hat{\pi}(x) t=\hat{\pi}(y) s$ and $x_{0}=y_{0}(s-t), y_{0}=x_{0}(t-s) \in W \cap Y=Z$. If $0<t \leqq s$, then $s-t<$ 
$t\left(y_{0}\right)$ implies that $s=t$. Thus, $x_{0}=y_{0}$ and $x=y$, since $x_{0}, y_{0} \in Z$. Thus $(x, t)=(y, s)$. Finally, the fact that $\hat{Z} \subset \pi^{-1}(W)$ implies that $\hat{Z} \boldsymbol{R} \subset \pi^{-1}(W)$ and $\hat{Z} \boldsymbol{R}$ being dense in $\hat{X}_{\hat{t}}$ proves that $\pi^{-1}(W)$ is dense.

If $X$ is one-dimensional, then (iv) of Lemma 2.5 implies one can choose $Y$ such that $\hat{X}=\left\{\left(\phi^{k}(x)\right)_{-\infty}^{\infty} ; x \in Y\right\}$. It follows that for $w \in$ $\hat{X}, \hat{t}(w)=t\left(w_{0}\right)$, and thus $\pi$ clearly induces an isomorphism between $\left(\hat{X}_{\hat{\imath}}, \boldsymbol{R}\right)$ and $(X, \boldsymbol{R})$.

3. Lifting $\mathscr{F}$-expansiveness. In this section we show that the model constructed in section two is a good one from the point of view of $\mathscr{F}$-expansiveness. In fact if $(X, \boldsymbol{R})$ is $\mathscr{F}$-expansive, then $\left(\hat{X}_{\hat{t}}, \boldsymbol{R}\right)$ is $\mathscr{F}$-expansive. We also obtain the results of Bowen and Walters in [2] for $\mathscr{C}$-expansive flows as a special case.

We first note that several other dynamical properties are lifted.

Proposition 3.1. (i) If $x \in \hat{X}$ and there is an integer $l$ such that $\varphi^{l}\left(x_{0}\right) \in S^{*}$ for some $S \in \mathscr{S}$, then $x_{j}=\varphi^{l}\left(x_{0}\right)$ for some integer $j$.

(ii) $x \in \hat{X}_{\hat{t}}$ is a transitive point if and only if $\pi(x) \in X$ is a transitive point.

(iii) $\left(\hat{X}_{\hat{t}}, \boldsymbol{R}\right)$ is minimal if and only if $(X, \boldsymbol{R})$ is minimal.

Proof. (i) For some $t \in \boldsymbol{R}, x_{0} t=\phi^{l}\left(x_{0}\right)$. Choose $\left\{z^{(n)}\right\} \subset \hat{Z}$ with $z^{(n)} \rightarrow x$ and $\left\{\alpha_{n}\right\}$ a sequence with $0<\alpha_{n} \leqq \alpha$ and $\alpha_{n} \rightarrow 0$. Then there is an increasing subsequence $\left\{M_{n}\right\}$ such that $z_{0}^{\left(M_{n}\right)}\left(t+\eta_{n}\right) \in S^{*}$, for some $S \in \mathscr{S}$ and $\eta_{n}$ with $\left|\eta_{n}\right|<\alpha_{n}$. Thus $z_{0}^{\left(M_{n}\right)}\left(t+\eta_{n}\right)=\phi^{j_{n}}\left(z_{0}^{\left(M_{n}\right)}\right)$ for some sequence $\left\{j_{n}\right\}$, and $\left|t+\eta_{n}\right| \leqq|t|+\alpha$ for all $n$ so $\left|j_{n}\right| \rho \leqq|t|+\alpha$ which implies $\left\{j_{n}\right\}$ is bounded. By choosing a subsequence if necessary assume $j_{n}=j$. Now $z_{0}^{\left(M_{n}\right)}\left(t+\eta_{n}\right)=\varphi^{j}\left(z_{0}^{\left(M_{n}\right)}\right)=z_{j}^{\left(M_{n}\right)} \rightarrow x_{j}$ but $z_{0}^{\left(M_{n}\right)} t \rightarrow \varphi^{l}\left(x_{0}\right)$. Thus $x_{j}=\varphi^{l}\left(x_{0}\right)$.

(ii) It suffices to show that $x$ is transitive when $\pi(x)$ is transitive. We can assume $x=(\hat{x}, 0) \in X$ and it is enough to show that $\hat{x}$ has a dense orbit in $(\hat{X}, \sigma)$. Choose $\left\langle U_{-n}, \cdots, U_{n}\right\rangle$ with each $U_{i} \subset S^{*}$, for some $S \in \mathscr{S}$, and $\left\langle U_{-n}, \cdots, U_{n}\right\rangle \cap \hat{X} \neq \phi$. By continuity of $\varphi^{i},|i| \leqq n$, at $\hat{w}_{0}$ where $\hat{w} \in\left\langle U_{-n}, \cdots, U_{n}\right\rangle \cap \hat{X}$, we can find an open $V \subset U_{0}$ such that $\mathrm{X}_{-n}^{n} \varphi^{i} V \subset\left\langle U_{-n}, \cdots, U_{n}\right\rangle$. Since $\hat{x}_{0}=\pi(x)$ is transitive under $\varphi$ in $Y$ we can find a $k$ with $\phi^{k} \hat{x}_{0} \in V$ and this point is $\hat{\pi}\left(\sigma^{j} \hat{x}\right)$ for some $j$ by (i ). Thus $\left(\sigma^{j} \hat{x}\right)_{i} \in U_{i}$ for $|i| \leqq n$, because $\varphi$ is continuous at $\left(\sigma^{j} \hat{x}\right)_{i}$ for $|i| \leqq n-1$, and so $\sigma^{j} \hat{x} \in$ $\left\langle U_{-n}, \cdots, U_{n}\right\rangle$.

(iii) Follows immediately from (ii).

It is not true in general that if $\pi(x)$ is almost periodic then $x$ is almost periodic. Simple counter examples can be constructed even in the codimension one case by using the flow on the 2-torus induced by a nonminimal homeomorphism of the circle without 
periodic points.

Recall that $(X, R)$ is topologically weak mixing if the product flow $(X \times X, \boldsymbol{R})$ has a transitive point.

Proposition 3.2. If $(X, \boldsymbol{R})$ is topological weak mixing (strong mixing) then $\left(\hat{X}_{\hat{t}}, \boldsymbol{R}\right)$ is weak mixing (strong mixing) also.

Proof. Suppose $(X, \boldsymbol{R})$ is weak mixing. Let $x, y \in Z$ such that $\left(x s_{1}, y s_{2}\right)$ has a dense orbit in $(X \times X, R)$. It is sufficient to show that for any choice of nonempty basic open sets $\left(\left\langle U_{-n}, \cdots, U_{n}\right\rangle \cap\right.$ $\hat{X})(-\varepsilon, \varepsilon) w_{1}$ and $\left(\left\langle V_{-n}, \cdots, V_{n}\right\rangle \cap \hat{X}\right)(-\varepsilon, \varepsilon) w_{2}$, where $\varepsilon>0, w_{1}, w_{2} \in \boldsymbol{R}$ and each $U_{i} \subset S_{0}^{*}$ for some $S_{0} \in \mathscr{S}$ and $V_{i} \subset S^{*}$ for some $S \in \mathscr{S}$, we can find $t \in \boldsymbol{R}$ with $(\hat{x}, 0)\left(s_{1}+\left(t-w_{1}+\eta_{1}\right)\right) \in\left\langle U_{-n}, \cdots, U_{n}\right\rangle$ and $(\hat{y}, 0)\left(s_{2}+\left(t-w_{2}+\eta_{2}\right)\right) \in\left\langle V_{-n}, \cdots, V_{n}\right\rangle$ for some $\left|\eta_{1}\right|,\left|\eta_{2}\right|<\varepsilon$. As in 3.1 we can find open $U, V \subset Y$ with $X_{-n}^{n} \varphi^{i} U \subset\left\langle U_{-n}, \cdots, U_{n}\right\rangle$ and $\mathbf{X}_{-n}^{n} \varphi^{i} V \subset\left\langle V_{-n}, \cdots, V_{n}\right\rangle$. Now choose $t$ such that $x s_{1}\left(t+\gamma_{1}-w_{1}\right) \in U$ and $y s_{2}\left(t+\gamma_{2}-w_{2}\right) \in V$ for some $\left|\gamma_{1}\right|<\varepsilon$ and $\left|\gamma_{2}\right|<\varepsilon$. Then as $\pi$ is one-to-one over $Z R,(\widehat{x}, 0)\left(s_{1}+\left(t+\gamma_{1}-w_{1}\right)\right) \in\left\langle U_{-n}, \cdots, U_{n}\right\rangle$ and $(\hat{y}, 0)\left(s_{2}+\left(t+\gamma_{2}-w_{2}\right)\right) \in\left\langle V_{-n}, \cdots, V_{n}\right\rangle$ as required.

The statement for strong mixing follows similarly by choosing $U$ and $V$ as above, noting that for some $T>0$ and all $|t|>T$, $U(t+\delta) \cap V \neq \phi$ for some $|\delta|<\varepsilon$, and finding $z \in U(t+\delta) \cap V \cap Z$, because then $\hat{z} \in\left\langle U_{-n}, \cdots, U_{n}\right\rangle(t+\delta) \cap\left\langle V_{-n}, \cdots, V_{n}\right\rangle$ and thus $\left\langle U_{-n}, \cdots, U_{n}\right\rangle(-\varepsilon, \varepsilon)\left(w_{1}+t\right) \cap\left\langle V_{-n}, \cdots, V_{n}\right\rangle(-\varepsilon, \varepsilon) w_{2} \neq \phi$ for all $t$ with $|t|>T+\left|w_{1}-w_{2}\right|$. This completes the proof.

It is not the case that all dynamical properties lift. Indeed, minimal flows on the 2-torus (which lift to suspensions of subshifts by 4.2$)$ show that if $(X, \boldsymbol{R})$ is equicontinuous (resp. distal), ( $\left.\hat{X}_{\hat{t}}, \boldsymbol{R}\right)$ need not be equicontinuous (resp. distal). However, with these two properties, $(X, \boldsymbol{R})$ is pointwise almost periodic, and thus for every $\hat{x} \in \pi^{-1}(W), \pi^{-1}(\pi \hat{x})=\{\hat{x}\}$ and $\pi \hat{x}$ almost periodic implies that $\hat{x}$ is almost periodic. In other words, the almost periodic points are residual in $\hat{X}_{\hat{t}}$. Finally, it is clear that if $x \in W$ is periodic and $\pi(\hat{x})=x$, then $\hat{x}$ is periodic, or if $x$ is strongly recurrent $(x \in \alpha(x) \cap$ $\omega(x))$, then so is $\hat{x}$.

With regard to strict ergodicity, suppose $\mu$ is the only $\boldsymbol{R}$-invariant measure on $X$ and $\mu(W)=1$. If $\hat{\mu}$ is any invariant measure on $\hat{X}_{\hat{t}}$, then $\pi(\hat{\mu})=\mu$ yields that $\hat{\mu}\left(\pi^{-1} W\right)=1$, and thus $\pi:\left(\hat{X}_{\hat{t}}, \hat{\mu}\right) \widetilde{\rightarrow}$ $(X, \mu)$ is a measure isomorphism. This together with 3.1 yields that $\left(\hat{X}_{\hat{t}}, \boldsymbol{R}\right)$ is strictly ergodic. However, it appears difficult to determine when the condition $\mu(W)=1$ holds in general. One special case where it does hold is when $(X, \boldsymbol{R})$ has codimension 1 , i.e., sections can be taken homeomorphic to the unit interval. In this case, 
$\mathscr{C}(Z)$ is countable (see 4.1) and $W^{\prime}=\mathscr{C}(Z) \boldsymbol{R}=\bigcup_{-\infty}^{\infty} \mathscr{C}(Z)[n, n+1)$. If $\mu\left(W^{\prime}\right)=1$, then for some $n, \mu(\mathscr{E}(Z)[n, n+1))>0$, and thus for some $w \in \mathscr{C}(Z), \mu(w[n, n+1))>0$. But this contradicts that $\mu$ is finite, noting that $(X, \boldsymbol{R})$ is minimal and hence not periodic.

We now turn to the expansive properties of $\left(\hat{X}_{\hat{t}}, \boldsymbol{R}\right)$.

Lemma 3.3. Suppose $x, y \in \hat{X}$ with $x \neq y$ and $\hat{\pi}(x)=\hat{\pi}(y)$. Then for some $n, x_{n}$ and $y_{n}$ belong to different sections.

Proof. $x_{0}=y_{0}$. Suppose that $n$ is the first integer such that $x_{n} \neq y_{n}$; assume $n>0$. (A similar argument covers $n<0$.) Now let $t=\hat{t}\left(\sigma^{n-1} x\right)$ and $s=\hat{t}\left(\sigma^{n-1} y\right)$. Then $x_{n}=x_{n-1} t$ and $y_{n}=y_{n-1} s$ and so $x_{n}(s-t)=y_{n}$. Since $|s-t| \leqq 2 \alpha<\zeta, x_{n}$ and $y_{n}$ can not belong to the same section.

Suppose that every pair of distinct points $x, y \in \hat{X}$ have the property that $x_{i}$ and $y_{2}$ belong to different sections for some $i$. Let $X(A)$ be the symbol space with $A=\{1,2, \cdots$, card $(\mathscr{S})\}$, (here we order $\mathscr{S}$ first), and define a map $\tau$ from $(\hat{X}, \sigma)$ into $(X(A), \sigma)$ by $\tau(x)_{i}=k$ where $x_{i}$ belongs to the $k$ th element of $\mathscr{S}$. It is easy to check that $\tau$ is an isomorphism from $(\hat{X}, \sigma)$ onto a subflow $\left(X^{\prime}, \sigma\right)$ of $(X(A), \sigma)$. Furthermore if we define $f$ on $X^{\prime}$ by $f(x)=\hat{t}\left(\tau^{-1} x\right)$ then clearly $\left(\hat{X}_{\hat{t}}, \boldsymbol{R}\right)$ is isomorphic to $\left(X_{f}^{\prime}, \boldsymbol{R}\right)$. Thus in this case $\left(\hat{X}_{\hat{t}}, \boldsymbol{R}\right)$ is a suspension over a subshift on a finite symbol space (and it is $\mathscr{C}$-expansive via Theorem 3.6 or directly as in Theorem 6 of [2]). With these comments we can now show that our construction generalizes the symbolic dynamics of [2].

Corollary 3.4. Suppose $(X, \boldsymbol{R})$ is real-expansive. Then we can choose $\mathscr{S}$ in such a way that for every pair of distinct points $x, y \in \hat{X}$ there is an integer $n$ such that $x_{n}$ and $y_{n}$ belong to different sections. Thus $(X, \boldsymbol{R})$ is the homomorphic image of a suspension over a subshift of a finite symbol space and the natural homomorphism is one-to-one between invariant residual sets. Furthermore if $X$ is one-dimensional, $(X, \boldsymbol{R})$ is (isomorphic to) this suspension.

Proof. Let $\zeta$ be as in Theorem 1.5 and let $\delta>0$ be a $\mathscr{C}$ expansive constant corresponding to $\zeta$. Choose $\alpha>0$ such that if $x, y \in X$ with $d(x, y) \leqq \alpha$ then $d(x s, y t)<\delta$ for $s, t \in \boldsymbol{R}$ such that $|s| \leqq \alpha$ and $|t| \leqq \alpha$. Choose a collection of sections $\mathscr{S}$ satisfying the conditions of Theorem 1.5 for this $\alpha$. Now suppose $x, y \in \hat{X}$ are such that $x_{i}$ and $y_{i}$ belong to the same section for every integer $i$ but $x \neq y$. Then $x_{0} \neq y_{0}$ by Lemma 3.3 and, since they belong to the same section, $x_{0} \notin y_{0}[-\zeta, \zeta]$ and $d\left(x_{0}, y_{0}\right) \leqq \alpha$. Now define a piecewise linear increasing function $f$ from $R$ into $\boldsymbol{R}$ such that 
$f(0)=0$ and $f\left( \pm \sum_{i=0}^{n} \hat{t}\left(\sigma^{i} x\right)\right)= \pm \sum_{i=0}^{n} \hat{t}\left(\sigma^{i} y\right)$, \pm determined by $\operatorname{sgn}(n)$. Then for any $t \in \boldsymbol{R}, d\left(x_{0} t, y_{0} f(t)\right)=d\left(x_{n} a, y_{n} b\right)$ where $t= \pm \sum_{i=0}^{n-1} \hat{t}\left(\sigma^{i} x\right) \pm$ $a, 0 \leqq a<\hat{t}\left(\sigma^{n} x\right)$ and $f(t)= \pm \sum_{i=0}^{n-1} \hat{t}\left(\sigma^{i} y\right) \pm b, 0 \leqq b<\hat{t}\left(\sigma^{n} y\right)$. Thus since $a \leqq \alpha$ and $b \leqq \alpha, d\left(x_{n} a, y_{n} b\right)<\delta$ and thus $d\left(x_{0} t, y_{0} f(t)\right)<\delta$ for all $t \in \boldsymbol{R}$ which is a contradiction.

In passing, one should note that the subflow $\left(X^{\prime}, \sigma\right)$ defined above can be defined without any assumption on $\hat{X}$, and $\tau$ induces a homomorphism from $(\hat{X}, \sigma)$ onto $\left(X^{\prime}, \sigma\right)$. Lemma 3.3 asserts that if $\hat{\pi}$ is not a homeomorphism, $X^{\prime}$ is nontrivial. Corollary 3.4 shows that $\mathscr{C}$-expansive implies that $\tau$ is an isomorphism; however, minimal flows on the two-torus show that $\tau$ can be an isomorphism without $\mathscr{C}$-expansiveness (see 4.2).

Before showing that $\mathscr{F}$-expansion lifts, we note a useful metric on a suspension of a flow $(W, \psi)$ induced by $g$, which we will utilize. If $0 \leqq t<1, x, y \in W$, set $d((x, t g(x)),(y, t g(y)))=(1-t) D(x, y)+$ $t D(\psi(x), \psi(y))$, where $D$ is the metric on $W$. Locally, distance along an orbit is normalized to 1 , i.e., $d((x, a),(x, b))=|a-b| / g(x)$. This extends to the whole suspension by taking the infimum of lengths of all "chains" along orbits and across orbits between two points. Finally, we use the metric $D(x, y)=\max \left[d_{0}\left(x_{i}, y_{i}\right) /|i|+1\right]$ on $\hat{X}$, where $d_{0}$ is the metric on $X$.

Lemma 3.5. Suppose that $x, y \in \hat{X}$ and $x_{i}$ and $y_{i}$ belong to different sections for some integer $i$. Then for any $u, v \in \boldsymbol{R}$ such that $-\hat{t}\left(\sigma^{-1} x\right)<u<\hat{t}(x),-\hat{t}\left(\sigma^{-1} y\right)<v<\hat{t}(y)$ and $|u-v|<\rho / 2$ and each $f \in \mathscr{C}$ there is $t \in \boldsymbol{R}$ with $d((x, 0)(u+t),(y, 0)(v+f(t))) \geqq \rho / 2$.

Proof. Suppose that $d((x, 0)(u+t),(y, 0)(v+f(t)))<\rho / 2$ for all $t \in \boldsymbol{R}$ and suppose $i>0$. (The proof for $i<0$ is similar.) Then for every $t \in \boldsymbol{R}$ we must be able to write $u+t=\sum_{j=0}^{n-1} \hat{t}\left(\sigma^{j} x\right)+a$ and $v+f(t)=\sum_{j=0}^{n-1} \hat{t}\left(\sigma^{j} y\right)+b$ where $n$ is the same for both sums, $|a-b|<\rho / 2$ and at least one of $a$ and $b$ is nonnegative. Now choose $t$ such that the corresponding $n$ yields that $x_{n}$ and $y_{n}$ belong to different sections and $a=0$. Then $d((x, 0)(u+t),(y, 0)(v+f(t)))=$ $d\left(\left(\sigma^{n} x, 0\right),\left(\sigma^{n} y, 0\right) b\right) \geqq 1 / 2 D\left(\sigma^{n} x, \sigma^{n} y\right) \geqq 1 / 2 \rho$, because $|b|<\rho / 2$ so that either $0 \leqq b<1 / 2 \hat{t}\left(\sigma^{n} y\right)$ or $-1 / 2 \hat{t}\left(\sigma^{n-1} y\right)<b<0$.

Theorem 3.6. Suppose $(X, \boldsymbol{R})$ is $\mathscr{F}$-expansive for some subset $\mathscr{F}$ of $\mathscr{C}$. Then $\left(\hat{X}_{\hat{t}}, \boldsymbol{R}\right)$ is $\mathscr{F}$-expansive also.

Proof. Let $\varepsilon<\rho / 2$ and let $\delta_{1}>0$ be a corresponding $\mathscr{F}$-expansive constant for $(X, \boldsymbol{R})$. Let $d_{0}$ be a metric on $X$. Now let $\delta>0$ be such that if $d_{0}(\pi(x, s), \pi(y, t))>\delta_{1}$ then $d((x, s),(y, t))>\delta$. Choose $(x, s),(y, t) \in \hat{X}_{\hat{t}}$ such that $(x, s) \notin(y, t)(-\varepsilon, \varepsilon)$. If $\pi(x, s) \notin$ 
$\pi(y, t)(-\varepsilon, \varepsilon)$ then for each $f \in \mathscr{F}$ there is $T \in \boldsymbol{R}$ such that $d_{0}(\pi(x, s) T$, $\pi(y, t) f(T))=d_{0}(\pi[(x, s) T], \pi[(y, t) f(T)])>\delta_{1} \quad$ and $\quad$ so $\quad d((x, s) T,(y$, t) $f(T))>\delta$. If $\pi(x, s) \in \pi(y, t)(-\varepsilon, \varepsilon)$, then $\pi(x, s)=\pi(y, t) \eta$ with $|\eta|<\varepsilon$. Assume $t \geqq s$, then $-\rho / 2<t-s+\eta \leqq \hat{t}(y)+\rho / 2$ and $x_{0}=$ $y_{0}(t-s+\eta)$ implies that $0 \leqq t-s+\eta \leqq \hat{t}(y)$. We now have three cases: (i) $x_{0}=y_{0}$, (ii) $x_{0}=y_{1}$ and (iii) neither of these in which case $x_{0}$ is a point on the $\varphi$ orbit of $y_{0}$ strictly between $y_{0}$ and $y_{1}$.

(i) If $x_{0}=y_{0}$, then since $|t-s+\eta|<2 \alpha<\zeta, t-s+\eta=0$ and thus $|t-s|<\rho / 2$ and so either $x=y$, or Lemmas 3.3 and 3.5 imply that we can find $T \in \boldsymbol{R}$ with $d((x, s) T,(y, t) f(T)) \geqq \rho / 2$. But $x=y$ is impossible, since then $(x, s)=(y, t) \eta \in(y, t)(-\varepsilon, \varepsilon)$ which is false.

(ii) If $x_{0}=y_{1}$ then $t-s+\eta=\hat{t}(y)$ and $\hat{\pi}(x)=\hat{\pi}(\sigma y)$ so that either $x=\sigma y$ or Lemma 3.3 implies that $x_{i}$ and $(\sigma y)_{i}$ belong to different sections for some integer $i$. But if $x=\sigma y$ then $(x, s)=$ $(y, 0)(s+\hat{t}(y))=(y, t) \eta \in(y, t)(-\varepsilon, \varepsilon)$ which is false. Now $\mid s-(t-$ $\hat{t}(y))|=| s+\hat{t}(y)-t|=| \eta \mid<\rho / 2$ so that by Lemma 3.5 we can find $T \in \boldsymbol{R}$ such that $d((x, 0)(s+T),(\sigma(y), 0)(t-\hat{t}(y)+f(T)))=d((x, s) T$, $(y, t) f(T)) \geqq \rho / 2$.

(iii) $x_{0}=y_{0}(t-s+\eta)$ and $|t-s+\eta|<\zeta$, so $x_{0}$ and $y_{0}$ are in different sections. Also, $D(x, y) \geqq \rho, D(\sigma x, \sigma y) \geqq \rho / 2$, and since $x_{0}$ and $y_{1}$ belong to different sections, $D(x, \sigma y) \geqq \rho$. Finally since $t-s+\eta<\hat{t}(y), x_{0}=y_{-1} v$ for some $v$ with $0<v<2 \alpha$, so that $x_{0}$ and $y_{-1}$ belong to different sections and thus $D(\sigma x, y) \geqq \rho / 2$. Thus we always have $d((x, s),(y, t)) \geqq \rho / 2$ for $0 \leqq s<\hat{t}(x)$ and $0 \leqq t<\hat{t}(y)$.

Thus $\min (\delta, \rho / 2)$ is an $\mathscr{F}$-expansive constant for $\left(\hat{X}_{\hat{t}}, \boldsymbol{R}\right)$ corresponding to $\varepsilon$, completing the proof.

4. Codimension one real flows. In this section we will use the special nature of sections in codimension one flows to obtain a geometric realization of the flow $\left(\hat{X}_{\hat{t}}, \boldsymbol{R}\right)$ in this case and to point out a further property of this model for minimal flows.

$(X, \boldsymbol{R})$ will be said to be codimension one if it has no fixed points and if all the local sections can be taken to be homeomorphic to $[0,1] . \quad(X, R)$ is codimension one if $X$ is a 2-manifold and the action is locally free and differentiable. This gives us a situation to which the results of [2] do not apply; it is shown in [3] that there are no $\mathscr{C}$-expansive flows on two manifolds. Indeed if $X$ is an orientable compact metric space minimal under $\boldsymbol{R}$, then it is a suspension over the circle ([7]) and so it can not be $\mathscr{C}$-expansive but it will exhibit various types of $\mathscr{F}$-expansiveness as discussed in [5].

The Model 4.1. The section arguments are similar to those of 
section two so we will only sketch the proofs. Choose $\mathscr{S}=$ $\left\{S_{1}, S_{2}, \cdots, S_{m}\right\}$ to be a collection of unit intervals i.e., $S_{i} \cong[0,1]$ and $S_{i}^{*} \cong(0,1)$. Let $\varphi$ be the first return map and $t$ be first return time as before. Set $A_{j k}=\left\{x \in S_{j}^{*} ; \varphi(x) \in S_{k}^{*}\right\}$, then $\varphi$ and $t$ are continuous on $A_{j k}$. Let $F_{j}=\left\{x \in S_{j}^{*} ; \varphi(x)\right.$ is an endpoint of some $S \in \mathscr{S}$ \}. Each $F_{j}$ is a finite set. (Note that the set $A$ of $\S 2$ is just the $2 m$ endpoints so that here each $\varphi^{i} A$ is closed and nowhere dense). Now $S_{j}-\left(F_{j} \cup\right.$ (endpoints of $\left.\left.S_{j}\right)\right)=\bigcup_{k=1}^{m} A_{j k}$ and so each $A_{j k}$ is a union of a finite number of disjoint open intervals. Call the collection of all these intervals $\left\{I_{1}, I_{2}, \cdots, I_{n}\right\}$. In fact $(Y, \varphi)$ can be thought of as an interval exchange transformation on the collection permitting contractions and expansions (or piecewise monotonic). Now if $x \in F_{j}$ then $\lim _{y \downarrow x} \varphi(y)=\underline{\varphi}(y), \lim _{y \uparrow x} \varphi(y)=$ $\bar{\varphi}(y), \lim _{y \downarrow x} t(y)=\underline{t}(y), \lim _{y^{\uparrow} x} t(y)=\bar{t}(y)$ all exist and this also holds if $x$ is an endpoint of an interval. For suppose that $\left\{x_{n}\right\}$ and $\left\{y_{n}\right\}$ are sequences increasing to $x$ and that $\lim _{n \rightarrow \infty} x_{n}$ and $\lim _{n \rightarrow \infty} y_{n}$ exist. We can form the combined monotonic sequence $\left\{z_{n}\right\}$ and since we can regard $\varphi$ as a map from $(0,1)$ into $(0,1),\left\{\varphi\left(z_{n}\right)\right\}$ must also be monotonic for $n$ large enough. Thus $\left\{\varphi\left(z_{n}\right)\right\}$ converges and so $\lim _{n \rightarrow \infty} x_{n}=\lim _{n \rightarrow \infty} y_{n}$. The other proofs are similar. Furthermore if $x \in F_{j}$, then $\varphi(x) \in\{\varphi(x), \bar{\varphi}(x)\}$. For if we let $[a, b]$ be a small interval containing $x$, then for $\varepsilon>0,[a, b](t(x)-\varepsilon, t(x)+\varepsilon)$ is a connected set containing $\varphi(x)$ in its interior so that for some $\beta$ either $[\varphi(x), \beta]$ is contained in this set or $[\beta, \varphi(x)]$ is contained in it. It immediately follows that $\varphi(x)=\varphi(x)$ in the first case and $\vec{\varphi}(x)=\varphi(x)$ in the second. In fact, $\varphi$ is uniformly continuous on each $I_{j}$, and thus extends to a homeomorphism on $\bar{I}_{j}$.

We can now produce $\hat{X}$ constructively. If $x \in F_{1}$, we produce sequences $\left\{x_{j}^{+}\right\}$and $\left\{x_{j}^{-}\right\}$as follows. "Split" $x$ to create $x_{0}^{+}$and $x_{0}^{-}$. If $\varphi(x)=\varphi(x)$, set $x_{1}^{+}=\varphi(x)$ and don't define $x_{1}^{-}$at all and if $\varphi(x)=$ $\bar{\varphi}(x)$, set $x_{1}^{-}=\varphi(x)$ and don't define $x_{1}^{+}$at all. Given a positive integer $l$, suppose we have defined $\left\{x_{0}^{+}, x_{p(1)}^{+}, \cdots, x_{p(l)}^{+}\right\}$and $\left\{x_{0}^{-}\right.$, $\left.x_{q(1)}^{-}, \cdots, x_{q(l)}^{-}\right\}$where $p(l) \leqq l, q(l) \leqq l$ and $\{p(i)\}$ and $\{q(i)\}$ are increasing. If $z_{l+1}=\varphi^{l+1}(x) \in S^{*}$ for some $S \in \mathscr{S}$, "split" $z_{l+1}$ and set $p(l+1)=p(l)+1, q(l+1)=q(l)+1, x_{p(l+1)}^{+}=z_{l+1}^{+}, x_{q(l+1)}^{-(l)}=z_{l+1}^{-}$. If $z_{l+1}$ is an endpoint and $z_{l+1}=\underline{\varphi}\left(\varphi^{l} x\right)$, set $p(l+1)=p(l)+1, x_{p(l+1)}^{+}=$ $z_{l+1}$ and $q(l+1)=q(l)$; if $z_{l+1}=\bar{\varphi}\left(\varphi^{l} x\right)$, set $q(l+1)=q(l)+1, x_{q(l+1)}^{-}=$ $z_{l+1}$ and $p(l+1)=p(l)$. We can continue the construction in this way and by deleting repetitions we obtain sequences $\left\{x_{j}^{+}\right\}$and $\left\{x_{j}^{-}\right\}$. Repeat the process for the points of $F_{2}$ not on the orbit of any point in $F_{1}$ and so on. Note that if $\hat{\varphi}$ is the continuous map induced by $\varphi$ then $\hat{\varphi}\left(x_{k c}^{ \pm}\right)=x_{k+1}^{ \pm}$so that $\hat{\varphi}$ is still "first return". The resulting set $\hat{X}$ becomes a compact metric space if we choose a metric $d$ 
such that $d\left(x_{r(l)}^{+}, x_{s(l)}^{-}\right) \rightarrow 0$ as $l \rightarrow \infty$ where $r(l)$ and $s(l)$ are such that $x_{r(l)}^{+}$and $x_{s(l)}^{-}$are the "splits" of the same point. Intuitively, we have "cut" points to insure continuity of $\phi$. We can now define $\hat{\pi}: \hat{X} \rightarrow Y$ by $\hat{\pi}\left(x_{r(l)}^{+}\right)=\hat{\pi}\left(x_{s(l)}^{-}\right)=\varphi^{l}(x)$. Note that $\hat{\pi}$ is continuous, at most 2-to-1, and $\hat{\pi}\left(\hat{\varphi}\left(x_{0}^{+}\right)\right)=\varphi(x)=x t(x)$ and $\hat{\pi}\left(\hat{\varphi}\left(x_{0}^{--}\right)\right)=\phi^{k}(x)=x t_{k}(x)$ (where $t_{k}(x)$ is $k$ th return time) for some $k>1$ if $x \in \bigcup_{j=1}^{m} F_{j}$ and $\varphi(x)=\varphi(x)$, and similarly in the other case. Similarly we can extend first return time $t$ to a function $\hat{t}$ continuous on $\hat{X}, \hat{t}(x)=$ $t(x)$ if $x$ is not a split point and $\hat{t}(x)=t_{k}(\hat{\pi}(x))$ for some $k \geqq 1$ if $x$ is a split point. We can now form the suspension $\left(\hat{X}_{\hat{t}}, \boldsymbol{R}\right)$ and show that $(X, \boldsymbol{R})$ is a quotient as in 2.6. The key to establishing that this "split point" construction is, in fact, the model of section two is to note that $\hat{\pi}^{-1}(x)$ is one point when $\varphi$ is continuous along the orbit of $x$ in both settings.

The next proposition shows that the model may well add expansiveness which was not present in the original flow (i.e., the converse of Theorem 3.5 does not hold). In fact, in the minimal case, flows which are never $\mathscr{C}$-expansive always have $\mathscr{C}$-expansive models.

Proposition 4.2. If $(X, \boldsymbol{R})$ is a codimension one minimal flow, then $\left(\hat{X}_{\hat{t}}, \boldsymbol{R}\right)$ is a suspension of a minimal subshift.

Proof. $\left(\hat{X}_{\hat{t}}, \boldsymbol{R}\right)$ is minimal by Proposition 3.1. We will show that $\hat{X}$ is zero-dimensional and $(\hat{X}, \hat{\varphi})$ is expansive which gives the result. In view of the method of 4.1 and Theorem 6 of [7] we can see that we are producing a suspension of the appropriate Sturmian minimal flow.

Suppose $a, b$ are distinct points in a connected component of $\hat{X}$. Then $[a, b] \subset \hat{X}$, but since every point of $Y$ has a dense orbit under $\varphi$ we can find a split point $x$ such that $a<\phi^{k}(x)<b$, for some integer $k$, and since $z=\varphi^{k}(x)$ is not an endpoint, we have $a<z^{+}<$ $z^{-}<b$ which is a contradiction, showing zero-dimensionality.

Choose $0<\varepsilon<\rho$ such that if $a, b \in S_{i}$ and $d_{0}(a, b)<\varepsilon$, then there is at most one $c \in S_{i}$ with $\varphi(c)$ an endpoint of some section and $c$ lying between $a$ and $b$. Now let $x, y \in \hat{X}$ and suppose that $\hat{\pi}(x), \hat{\pi}(y) \in S_{j}$, some $j, \hat{\pi}(x)<\hat{\pi}(y)$ and $d_{0}(\hat{\pi}(x), \hat{\pi}(y))<\varepsilon$. Choose $z \in Y$ and $k>0$ such that $\hat{\pi}(x)<z<\hat{\pi}(y), \phi^{k}(z)$ is an endpoint of a section, and $k$ is minimal with respect to this property. The existence of such a point follows since every point of $Y$ has a dense orbit under $\varphi$. It now follows that for $0 \leqq j \leqq k-1, \varphi^{j}$ is continuous on $(\hat{\pi}(x), \hat{\pi}(y))$ and by the remarks of 4.1 we can extend $\varphi$ so that $\phi^{j}$ is continuous on $[\hat{\pi}(x), \hat{\pi}(y)]$. Then $w=\phi^{k-1}(z)$ lies between $\varphi^{k-1} \hat{\pi}(x)$ and $\varphi^{k-1} \hat{\pi}(y)$ and thus $d\left(\hat{\varphi}^{k-1} x, \hat{\varphi}^{k-1} y\right)>d\left(w_{0}^{+}, w_{0}^{-}\right)$. Finally, 
if $\quad 0<\varepsilon_{0}<\rho \quad$ satisfies $\quad d_{0}(\hat{\pi}(w), \hat{\pi}(v)) \geqq \varepsilon$ implies $d(w, v) \geqq \varepsilon_{0}$ then $\min \left\{\varepsilon_{0}, d\left(a_{0}^{+}, a_{0}^{-}\right) ; a \in \bigcup_{j=1}^{m} F_{j}\right\}$ is an expansive constant for $(\hat{X}, \hat{\varphi})$.

It would be interesting to generalize the results of [2] and of this paper to flows with other acting groups, for example $\boldsymbol{R}^{2}$. At present the authors know of no results along these lines.

\section{REFERENCES}

1. G. D. Birkhoff, Dynamical Systems, AMS Colloquium Publ., Vol. 9 Providence, R.I., 1966.

2. R. Bowen and P. Walters, Expansive one parameter flows, Differential Equations, 12 (1972), 180-193.

3. L. W. Flynn, Expansive flows on 2-manifolds a non-existence theorem, preprint.

4. H.B. Keynes and J. Robertson, Generators for topological entropy and expansiveness, Math. Sys. Theory, 3 (1969), 51-59.

5. H. B. Keynes and M. Sears, F -expansive transformation groups, General Topology and Its Applications, 10 (1979), 65-87.

6. S. Schwartzman, Global cross sections of compact dynamical systems, Proc. Nat. Acad. Sciences, 48 (1962), 786-791.

7. R. S. D. Thomas, Commuting continuous flows on manifolds, Math. Sys. Theory, 4 (1970), 193-197.

8. H. Whitney, Regular families of curves, Ann. of Math., 34 (1933), 244-270.

Received February 9, 1979. Research of the first author was supported by NSF Grant \#MCS 78-00403 and of the second author by a postdoctoral bursary from the CSIR.

UnIVERSITY OF MinNeSOTA

MiNNEAPOLIS, MN 55455 


\section{PACIFIC JOURNAL OF MATHEMATICS}

\section{EDITORS}

DONALD BABBITT (Managing Editor)

University of California

Los Angeles, California 90024

HUgo RossI

University of Utah

Salt Lake City, UT 84112

C. C. MOORE and ANDREW OGG

University of California

Berkeley, CA 94720

\section{J. DUGUNDJI}

Department of Mathematics University of Southern California Los Angeles, California 90007

R. Finn and J. Milgram Stanford University Stanford, California 94305

\section{ASSOCIATE EDITORS}

E. F. BECKENBACH

B. H. NEUMANN

F. WOLF

K. YoshidA

\section{SUPPORTING INSTITUTIONS}

UNIVERSITY OF BRITISH COLUMBIA CALIFORNIA INSTITUTE OF TECHNOLOGY UNIVERSITY OF CALIFORNIA MONTANA STATE UNIVERSITY UNIVERSITY OF NEVADA, RENO NEW MEXICO STATE UNIVERSITY OREGON STATE UNIVERSITY UNIVERSITY OF OREGON
UNIVERSITY OF SOUTHERN CALIFORNIA STANFORD UNIVERSITY UNIVERSITY OF HAWAII UNIVERSITY OF TOKYO UNIVERSITY OF UTAH WASHINGTON STATE UNIVERSITY UNIVERSITY OF WASHINGTON 


\section{Pacific Journal of Mathematics}

Vol. 85, No. $1 \quad$ September, 1979

Ralph Alexander, Metric averaging in Euclidean and Hilbert spaces...... 1

B. Aupetit, Une généralisation du théorème de Gleason-Kahane-Żelazko

pour les algèbres de Banach..............................

Lung O. Chung, Jiang Luh and Anthony N. Richoux, Derivations and

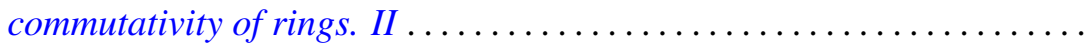

Lynn Harry Erbe, Integral comparison theorems for third order linear

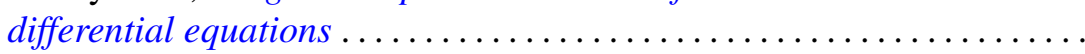

Robert William Gilmer, Jr. and Raymond Heitmann, The group of units of a

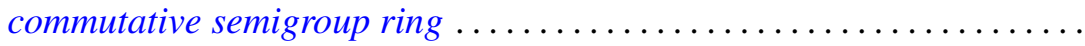

George Grätzer, Craig Robert Platt and George William Sands, Embedding lattices into lattices of ideals ...........................

Raymond D. Holmes and Anthony Charles Thompson, $n$-dimensional area and content in Minkowski spaces ....................... 77

Harvey Bayard Keynes and M. Sears, Modelling expansion in real flows....

Taw Pin Lim, Some classes of rings with involution satisfying the standard

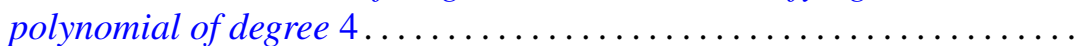

Garr S. Lystad and Albert Robert Stralka, Semilattices having bialgebraic congruence lattices ................................... 131

Theodore Mitchell, Invariant means and analytic actions . . . . . . . . . 145

Daniel M. Oberlin, Translation-invariant operators of weak type ........ 155

Raymond Moos Redheffer and Wolfgang V. Walter, Inequalities involving

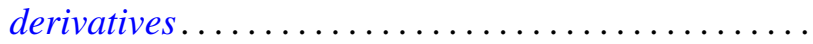

Eric Schechter, Stability conditions for nonlinear products and semigroups ................................

Jan Søreng, Symmetric shift registers ........................ 201

Toshiji Terada, On spaces whose Stone-Čech compactification is $\mathrm{Oz} \ldots \ldots .231$

Richard Vrem, Harmonic analysis on compact hypergroups ... 